\title{
Regularization of MR Diffusion Tensor Maps for Tracking Brain White Matter Bundles
}

\author{
C. Poupon ${ }^{1,2}$, J.-F. Mangin 1 , V. Frouin ${ }^{1}$, J. Régis ${ }^{3}$, \\ F. Poupon ${ }^{1}$, M. Pachot-Clouard ${ }^{1}$, D. Le Bihan ${ }^{1}$ and I. Bloch ${ }^{2}$ \\ 1 Service Hospitalier Frédéric Joliot, Commissariat à l'Energie Atomique, \\ 91401 Orsay Cedex, France, e-mail : cpoupon@shfj.cea.fr \\ 2 Ecole Nationale Supérieure des Télécommunications, 75013 Paris, France \\ 3 Service de Neurochirurgie Fonctionnelle et Stéréotaxique, \\ La Timone, Marseille, France
}

\begin{abstract}
We propose a new way for tracking brain white matter fiber bundles in diffusion tensor maps. Diffusion maps provide information about mobility of water protons in different directions. Assuming that diffusion is more important along axons, this information could lead to the direction of fiber bundles in white matter. Nevertheless, protocoles for diffusion image acquisition suffer from low resolutions and instrument noise. This paper is essentially dedicated to the design of a Markovian model aiming at the regularization of direction maps, and at the tracking of fiber bundles. Results are presented on synthetic tensor images to confirm the efficiency of the method. Then, white matter regions are regularized in order to enable the tracking of fiber bundles, which is of increasing interest in functional connectivity studies.
\end{abstract}

\section{Introduction}

During the last decade, the wide development of magnetic resonance imaging (MRI) has led to the design of a lot of segmentation methods dedicated to brain structures. For instance, the cortex, the white matter and the deep nuclei can be efficiently extracted from standard T1-weighted inversion-recovery MRI. Nevertheless, in spite of the $1 \mathrm{~mm}$ resolution reached by most of the MR sequences, the fiber bundles embeded in white matter can not be tracked.

Therefore, few image analysis methods have been dedicated to brain white matter. Skeletonization has been proposed in [1] to analyze its complex convoluted shape. Simulations of information transmission have been proposed in [2] to detect inter-hemispheric connections. However, white matter organization is of increasing interest in the neuroscience community. For instance, a tension-based mechanism has been recently hypothesized as an explanation of the cortex folding pattern [3]. Moreover, connectivity between different brain areas is a crucial information to infer the brain functional organization. The recent advent of very fast MR sequences, like the echo-planar technique, has opened a new field of modalities which give access to this kind of information. Diffusion imaging implies the acquisition of a great amount of images from which water mobility in different directions is inferred. Assuming that this mobility is more important along axons than in any other direction, the fiber direction can be computed for each voxel of the white matter. Hence, fiber bundles can be tracked throughout 
the whole brain white matter which is the scope of this paper.

In this paper, we try to explain what kind of information is contained in diffusion maps, and we propose a way of representing tensor information about fiber orientation. Second, we present a Markovian model designed to regularize fiber orientation images. Finally, the behavior of this model with synthetic and real data is described.

\section{Diffusion tensor imaging (DTI)}

\subsection{Diffusion process}

In brain tissues, molecules are submitted to a Brownian motion in time that is macroscopically a diffusion process. In the case of an isotropic liquid, the probability that a molecule moves from a distance $r$ during the time $t$ follows a Gaussian law with a variance $\operatorname{var}(r)=6 D t$ where $D$ is the diffusion coefficient that characterizes the mobility of molecules. In anisotropic environments, the mobility is different along each direction of space. Hence, diffusion is a tridimensional process which can be modeled by a second order tensor. The matrix $[D]$ is symmetric, positive and real, i.e represents a quadratic form. The diffusion coefficient in any direction $\vec{d}$ is given by the tensorial dot product (equation 1 ). $[D]$ is an intrinsic property of the tissue [4][5][6][7].

$$
\delta=\vec{d}^{T}[D] \vec{d}, \text { with }[D]=\left(\begin{array}{lll}
D_{x x} & D_{x y} & D_{x z} \\
D_{y x} & D_{y y} & D_{y z} \\
D_{z x} & D_{z y} & D_{z z}
\end{array}\right)
$$

\subsection{Contents of DTI : reading the raw data}

Acquisitions have been done on a GE SIGNA 1,5T. Imaging acquisition parameters were as follows: 52 axial slices, $3 \mathrm{~mm}$ slice thickness, $128 \times 128$ in planeresolution with a $1.875 \mathrm{~mm}$ size. The six coefficients of $[D]$ are represented in figure 1 for a slice. Some large bundles, such as the corpus callosum or the cortico-spinal fibers, are highlighted in the raw images because they are colinear with one image axis. For instance, an hypersignal (white zone) on the $D_{x x}$ coefficient slice shows an important diffusion process in the $\mathrm{x}$ axis, in the corpus callosum, whereas the $D_{y y}$ and $D_{z z}$ coefficient slices present a very low signal at the same location, revealing a lack of diffusion in these directions.

It must be noted that it is very hard to understand the information of the tensor by looking at images of the 6 coefficients of the tensor.

\subsection{Visualizing tensor information}

It is not easy to read simultaneously 6 images and it would be easier to understand the diffusion if they were gathered in one image. With this aim, we compute the eigen system $\left\{\left(\vec{e}_{1}, \lambda_{1}\right),\left(\vec{e}_{2}, \lambda_{2}\right),\left(\vec{e}_{3}, \lambda_{3}\right) / \lambda_{1}>\lambda_{2}>\lambda_{3}\right\}$ associated to the tensor $[D]$, for each voxel. A fiber bundle has a strong anisotropy, and a local tensor of that bundle has a first eigen value $\lambda_{1}$ much greater than the others. The diffusion of water protons is the most important along the direction $\vec{e}_{1}$. A basic idea to represent the information contained in a tensor map is to detect the strongly anisotropic voxels and to represent, in such voxels, the eigen vector $\vec{e}_{1}$ by a cylinder. An alternative is to represent an ellipsoid, the axis of which have lengths equal to the 3 eigenvalues of the tensor. But, the visualization in 


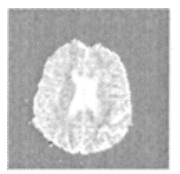

Dxx

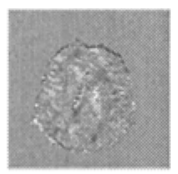

Dxy

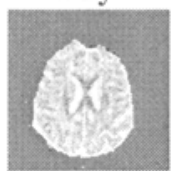

Dyy

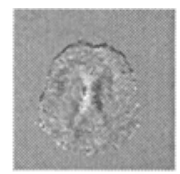

Dxz

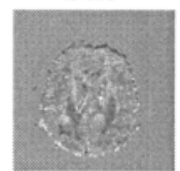

Dyz

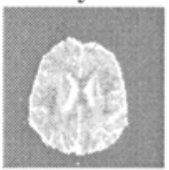

Dzz

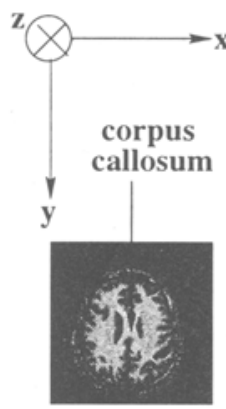

Anatomy

(T1-weighted slice)

Diffusion tensor images

Fig. 1. Slices of a brain diffusion tensor map; the 6 coefficients are represented, and a T1 MRI gives the corresponding anatomy.

3D is more complicated for a large set of ellipsoïds. Several factors of anisotropy can be found in the litterature [8][6], but we have decided to choose a basic one (equation 2). A fully anisotropic tissue has a factor 1.0 since $\operatorname{trace}[D]=\lambda_{1}$, and an isotropic tissue has a factor 0.0 since $\lambda_{1}=\lambda_{2}=\lambda_{3}$.

$$
a=\frac{3}{2}\left(\frac{\lambda_{1}}{\operatorname{trace}([D])}-\frac{1}{3}\right)
$$

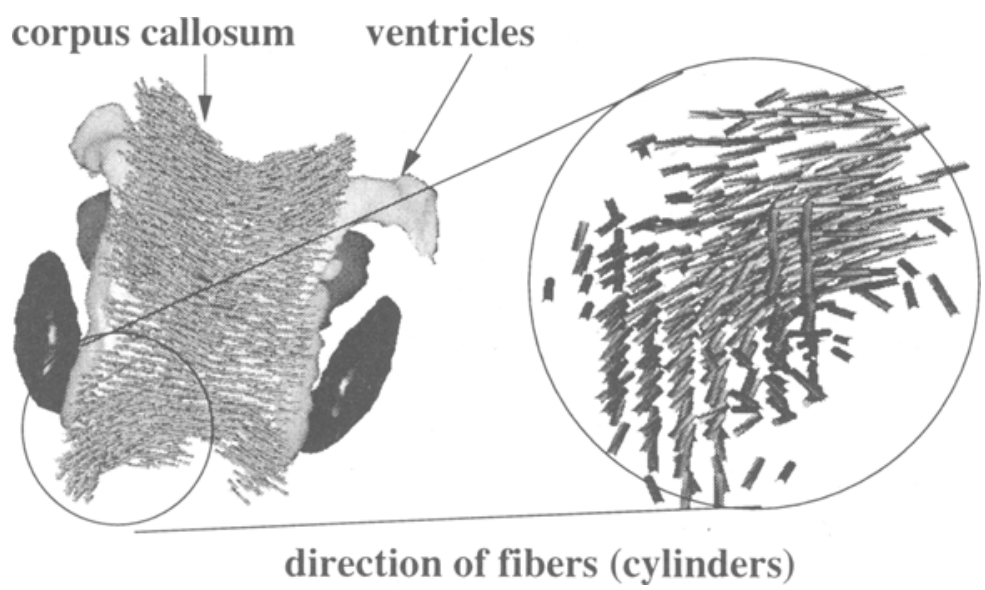

Fig. 2. Representation of the main directions of diffusion with small cylinders

Figure 2 gives a result of that simple method applied to a volume of interest around the corpus callosum. Each direction of fiber is represented by a small cylinder which axis is colinear to $\overrightarrow{e_{1}}$ direction and which length is proportional to the anisotropy factor. A threshold on anisotropy has been done to get only directions of the most anisotropic tissues. We clearly observe the corpus callosum. 
We have inserted the deep nuclei and the ventricles in this image to help its understanding (anatomical structures have been segmented with a region growing method). A zoom on the corpus callosum shows that this image of vectors is not very noisy, but in other white matter areas which are less anisotropic, the noise is more important and must be corrected.

\subsection{Origins of the noise in DTI}

Two main reasons may explain the noise in diffusion tensor maps. A first source of artifacts can be found in the motion of the ventricles. The measurements of tensor are probably blurred by a periodic beat of the cerebro-spinal fluid (CSF) related to blood flow.

Second, partial volume effect blurs the estimation of the tensor, in the case where several fiber bundles cross into one simple voxel. Each fiber direction will favor diffusion along its own axis. This leads to a tensor much more difficult to interpret, which highlights the limit of the underlying linearization of the diffusion phenomenon. Consequently, the current resolution ( $3 \mathrm{~mm}$ thickness) will not allow the tracking of very thin bundles. All those sources of noise led us to develop a Markovian random field approach for regularizing maps of potential directions of bundles.

\section{Markovian regularization: the spaghetti plate model}

\subsection{Markovian assumption}

Let us consider a voxel of white matter where the fibers follow direction $\vec{d}$. Since fibers can not end up inside white matter, we have to find neighboring voxels, forwards and backwards, with similar fiber directions (or perhaps the boundary of white matter). Moreover, this property seems sufficient to define the whole geometry of white matter fiber directions. Therefore, we have designed Markovian models based on this property which will be used to regularize the fiber orientation image in a classical Bayesian framework [9]. Let $\vec{d}(M)$ denotes the putative fiber direction for voxel $\mathrm{M}$. The realizations of the random variable $\vec{d}(M)$ of the field are unitary vectors. Each variable state space corresponds to all directions of the $3 \mathrm{D}$ space.

We call this class of models the spaghetti plate models because the deep local minima of the underlying energy look really like this. Hence, the role of the data will be to choose the spaghetti plate corresponding to the underlying white matter geometry namely endowed with the highest proton mobility along spaghetti. In the following, we discuss different types of interaction potentials $V_{S}(\vec{d}(M))$ leading to spaghetti plate models.

The whole regularization model has to combine the a priori model on geometry with the data. The classical Bayesian approach leads to minimizing a sum of two terms:

$$
E=\sum_{M \in \text { Volume }} V_{S}(\vec{d}(M))+\alpha \sum_{M \in V \text { olume }} V_{D}(\vec{d}(M))
$$

where $V_{D}(\vec{d}(M))$ should be a function inversely proportional to the mobility of water protons in direction $\vec{d}(M)$ ( $\alpha$ is a weight). 


\subsection{Interaction potentials}

If we have a synthetic look at the global appeareance of the fiber bundles in the brain, one may think about a spaghetti plate. When not enough cooked, spaghetti stay pasted together and form some bundles of a few spaghetti. The spaghetti of a same bundle bend under the action of water. The whole packets of spaghetti leads to a plate of interlaced bundles. It is important to notice that a spaghetti not enough cooked cannot have a strong local curvature. Thus, when we move forward along the spaghetti, the direction $\vec{d}$ of the spaghetti can be only slightly modified. This property is used to give a mathematical expression to the spaghetti plate model. Let $\vec{d}(M)$ denotes the putative fiber direction for voxel $M$. When we consider the set of voxels $M$ of a direction map of spaghetti, we can write the global energy of the spaghetti configuration by adding all the local potentials (equation 4 ).

$$
E_{S}=\sum_{M \in \text { Volume }} V_{S}(\vec{d}(M))
$$

Let us consider the figure 3.1. For all the neighbors $\mathrm{N}$ of the voxel $\mathrm{M}$ contained in a solid angle $\beta$ (forward and backward) which axis is in the potential direction of spaghetti (or fibers) of the voxel $M$, we compute the angle $\theta(M, N)=(\vec{d}(M), \vec{d}(N))$. If all those neighbors $\mathrm{N}$ belong to the same bundle, then the angles $\theta(M, N)$ have to be small. The first idea consists in using all $\theta(M, N)$ to give an expression of the "spaghetti" potential $V_{S}(\vec{d}(M))$ at $\mathrm{M}$ (equation 5):

$$
V_{S}(\vec{d}(M))=\sum_{N \in \mathcal{V}(M) \cap N^{26}}\left|\cos ^{-1}(\vec{d}(M) \cdot \vec{d}(N))\right|
$$

where $\mathcal{V}(M)$ is a cone of apex $\mathrm{M}$, direction $\vec{d}(M)$ and aperture angle $\beta$, and $N^{26}$ is the 26-neighborhood. Using such a conic neighborhood discards the influence of voxels which could belong to bundles with other directions. The aperture $\beta$ of the solid angle which defines the neighborhood is set typically to $45^{\circ}$. In order to allow a range of quasi-equivalent potential for low angles, we finally used the angle cosine rather than a linear function (equation 6). Other choices could be investigated in the future.

$$
V_{S}(\vec{d}(M))=-\sum_{N \in \mathcal{V}(M) \cap N^{26}}|\cos (\theta(M, N))|
$$

Figure 3.2 shows an example of the generation of a spaghetti plate given by this model. The spaghetti directions have been initialized randomly. The energy made up by the sum of $V_{S}(\vec{d}(M))$ over the whole image has been minimized, using a deterministic algorithm. Voxels outside the image are discarded from the conic neighborhood, which means that no constrain on fiber direction is induced by white matter boundary. Three of the spaghetti have been isolated to point out their low local curvature.

Then, we have built a synthetic object to simulate the case of two noisy orthogonal bundles locally tangent. The image to process is given in figure 4.1a. A deterministic minimization of the energy stemming from the model 6 has been 


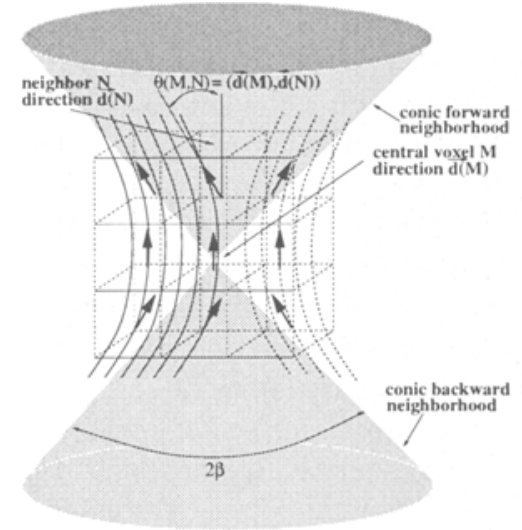

(1)

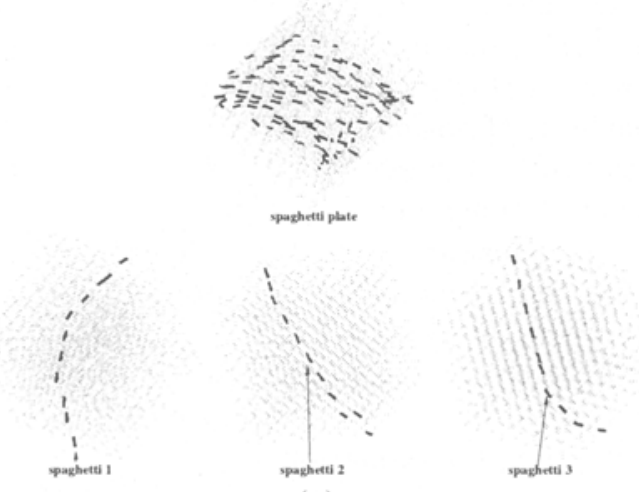

(2)

Fig. 3. (1) Schematic representation of the conic neighborhood of a voxel. (2) Generation of spaghetti by minimization of the energy corresponding to model 6 .

performed $\left(\beta=60^{\circ}\right)$ to get the image given in figure $4.1 \mathrm{~b}$. This example points out the weaknesses of the model. The model 6 considers all the neighbors in the cone. The effect of regularization and smoothing may be too important in the case of small bundles or at the interface of two large tangent bundles with very different orientations. This over-regularization effect can be overcome if a fixed number of neighbors is selected in both half-cones.

Let us write $\mathcal{V}^{b}(M)$, the subset of the neighborhood $\mathcal{V}(M)$ with the best neighbors:

$\mathcal{V}^{b}(M)=V_{\text {forward }}^{b}(M) \cup V_{\text {backward }}^{b}(M)$ where $V_{\text {forward }}^{b}(M)$ (respectively $\left.V_{\text {backward }}^{b}(M)\right)$ is made up by the b forward (respectively backward) 26-neighbors with lowest angles $\theta(M, N)$ (equation 7 ).

$$
V_{S}(\vec{d}(M))=-\sum_{N \in \mathcal{V}^{b}(M)}|\cos (\theta(M, N))|
$$

The same test as in figure 4.1 has been performed with $b=4$, leading to good results (figure 4.2 ).

The local minima of this energy are spaghetti plates, and there can be a huge number of configurations. Among these, we should be able to determine the closest configuration to the white matter geometry. That is the role of a second potential of attachment to the data.

\subsection{Data influence}

Indeed, we want to select the spaghetti plate with the highest global diffusion. It is possible, with the local tensor $[D(M)]$, to give the value of that diffusion in any direction $\vec{d}$, using the tensorial dot product (equation 1 ). Then we use equation 8 for the potential $V_{D}(\vec{d}(M))$ :

$$
V_{D}(\vec{d}(M))=-\vec{d}(M)^{T}[D(M)] \vec{d}(M)
$$




\subsection{Using anisotropy}

The spagheti model 7 docs not lake into account local variations of the anisotropy in the neighborhood of a voxel $\mathrm{M}$, so that a voxel $\mathrm{N}$ with nearly isotropic tensor acts upon its neighborhood, whereas this voxel $\mathrm{N}$ does not contain any fiber. One solution to avoid this problem is to weight each term of the potential $V_{S}(\vec{d}(M))$ corresponding to the neighbor $\mathrm{N}$ by the anisotropy $a(N)$ (equation 9 ). Tests on the synthetic image figure 4.3 a reveals that the effect is very important on the ridges of rectangular fibers (figure $4.3 \mathrm{~b}$ ), because these lines include few points of the bundle in their conic neighborhood. The anisotropy balancing clearly prevents such effects (figure 4.3c).

$$
V_{S}(\vec{d}(M))=-\sum_{N \in \mathcal{V}^{b}(M)} a(N)|\cos (\theta(N))|
$$

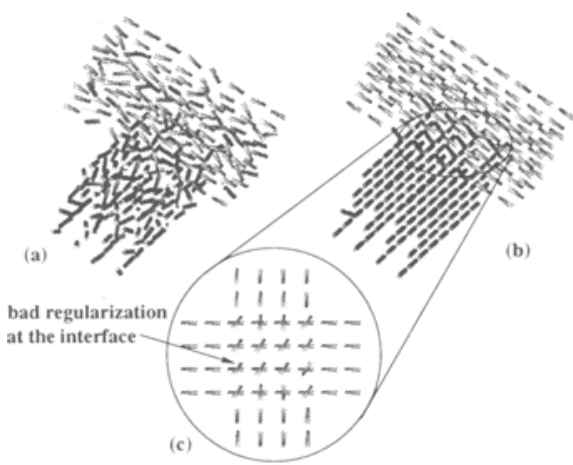

(1)

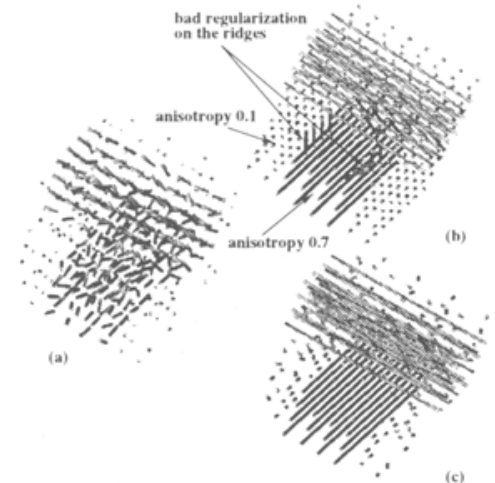

(3)

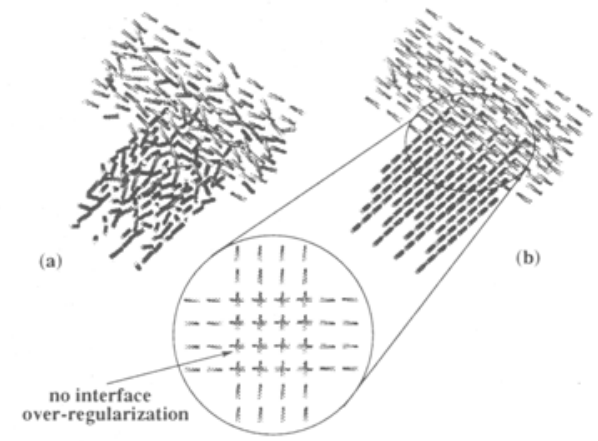

(2)

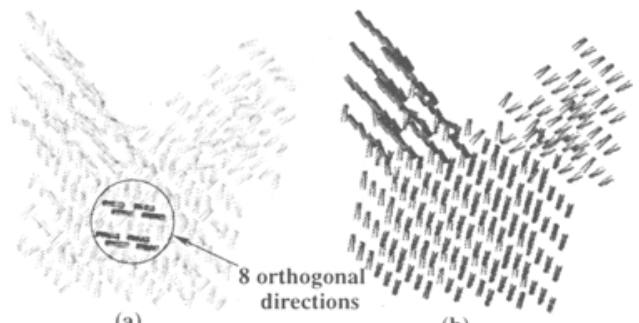

(a) (b)

Fig.4. (1) Over-regularization at the interface of 2 perpendicular bundles with model 6; (2)Result of energy minimization of the model 7 considering the 4 best neighbors in each half-cone; (3)Introduction of the anisotropy in the spaghetti model 9. (4) Synthetic tensor $Y$-shaped object with 8 arbitrary misplaced directions; (a) start image, (b) result of regularization with model 9 . 


\section{Results}

\subsection{Minimization of the energy}

The direction map of our Markovian random field has to be initialized not too far from the solution to speed-up the convergence. The direction of each voxel $\mathrm{M}$ is set to have the greatest diffusion, namely to the $\vec{e}_{1}(M)$ unit eigen vector direction computed from the tensor $[D(M)]$.

The space of variation of the directions $\vec{d}(M)$ is the $3 \mathrm{D}$ continous space. To be sure to try uniformly every direction of that space, we have sampled a unit sphere and we test, for each voxel M, all the sampled directions. The sampling of the unit sphere (typically 652 directions) is based on an iterative algorithm starting from an icosahedron and giving a uniform distribution of directions.

The global energy of the model is a linear combination of the "spaghetti" model energy and of the data attachment energy. We have to balance the influence of data by the weight $\alpha$. The choice of the factor $\alpha$ depends on the value of the mean trace in anisotropic areas, typically $2.0 \times 10^{-9}$ in white matter, and on the mean spaghetti energy. Since our initialization is not so far from the searched minimum of that energy $\mathbf{E}$, we used a deterministic ICM algorithm to minimize $\mathrm{E}[10]$.

\subsection{Synthetic image}

We have explicitly put 8 local directions in an $Y$ structure at $90^{\circ}$ of the direction of the bundle to see if the model corrects them efficiently (figure 4.4(a)). To simulate noisy data, we have modified each direction $\vec{d}(M)$ within a $60^{\circ}$ solid angle around $\vec{d}(M)$. The parameters of the model 9 with the 4 best neighbors in each cone are as follows: $\alpha=0.5, \beta=45^{\circ}, 652$ uniformly distributed directions. The bundle has an anisotropy of 0.7 whereas the environment has an anisotropy of 0.1 . After convergence of ICM, we get the result (figure 4.4(b)), where the directions are perfectly regularized.

\subsection{Brain white matter bundles}

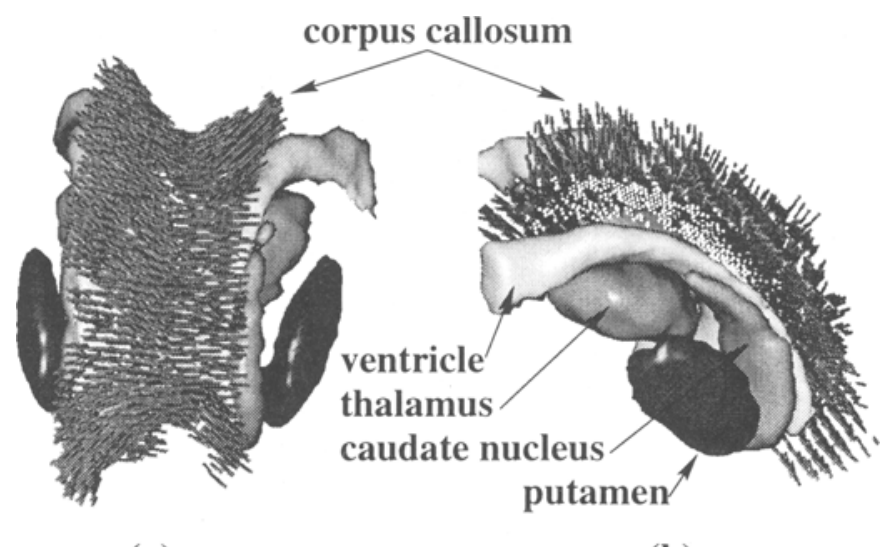

(a)

(b)

Fig. 5. Regularization of the corpus callosum

We have tested our algorithm on brain white matter to regularize fiber bundles.

We have chosen some regions of interest around the corpus callosum (figure 
5) and around the pyramidal pathways (6) which gather thalamo-parietal and cortico-spinal fibers. In such thick bundles, local angular deviation never overruns $30^{\circ}$, hence an aperture $\beta=45^{\circ}$ is sufficient to follow a fiber. The parameter $\alpha$ has been empirically set to $10^{9}$. For representing only anisotropic bundles, we have weighted the length of every cylinder (representing a fiber direction) by its anisotropy, as before. The mean anisotropy is 0.7 in the corpus callosum and 0.5 in the pyramidal bundle.

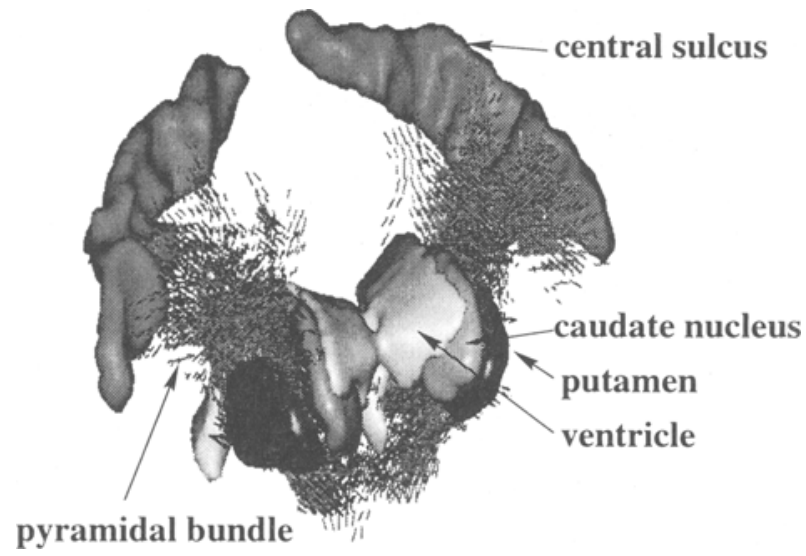

Fig. 6. Regularization of the pyramidal pathways

With such regularized images, we are now able to track a fiber direction. two points. For a given voxel M, we look forwards (respectively backwards) for the best neighbor $\mathrm{N}$ and we propagate throughout white matter. For instance, we have tracked one fiber direction of the pyramidal pathways. This direction belongs to the cortico-spinal buncle which goes from the primary motor areas inside precentral gyrus until the brain stem passing through the internal capsule (figure 7).

\section{Conclusion}

We have proposed a new method for tracking brain white matter fiber bundles in diffusion tensor maps. Diffusion maps provide the mobility of water proton in any direction of the 3D space. This information is used to determine the direction of maximum diffusion which is assumed to be that of a fiber bundle. However, diffusion maps are blurred by instrument noise and low resolution. In order to regularize them, we have developed a Markovian framework based on a spaghetti plate model.

Some tests on synthetic tensor images led us to improve the basic model to get a robust algorithm. Then, the regularization process has been implemented on a human brain image. It takes approximately 1 hour on a SPARC-ULTRA-30 station, but a reasonable computation time can be expected with a code-optimized version. Regions of interest around the corpus callosum and the pyramidal pathways have been selected to show results. Finally, an algorithm for tracking single fibers has been developed to give the best path of water diffusion from a given point.

In the future, we plan to correlate such information on brain connectivity with studies of functional connectivity using fMRI, which is a question of increasing interest in brain mapping studies. 


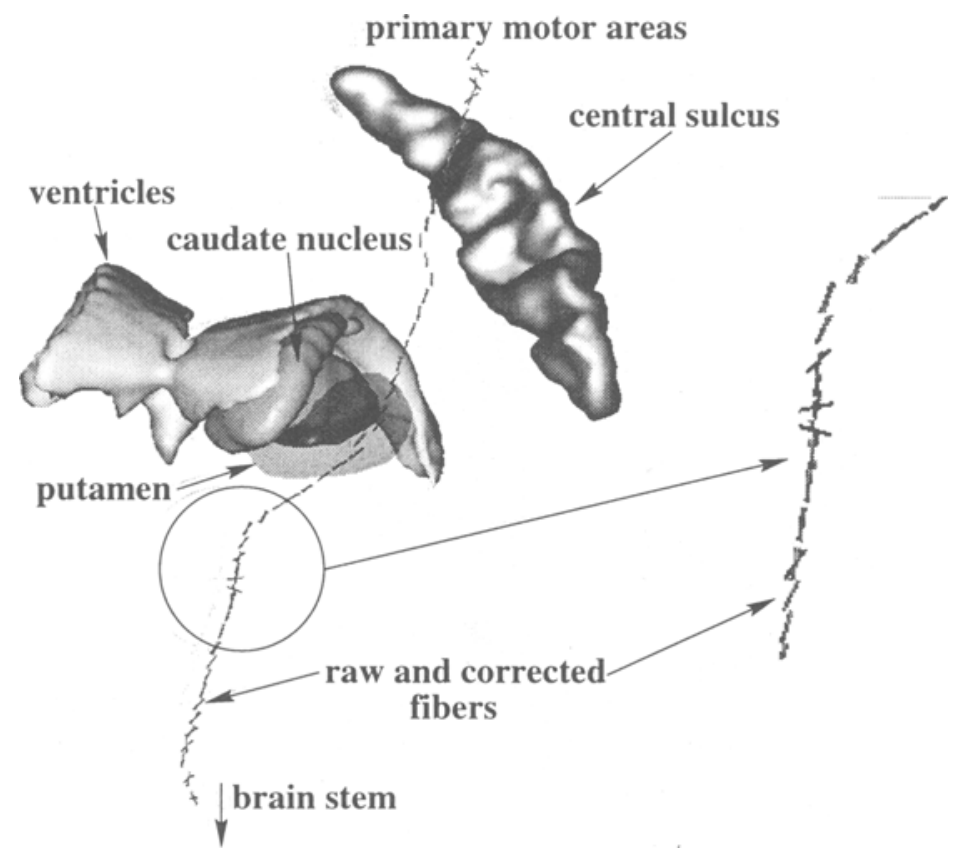

Fig. 7. The regularization allows the tracking of a fiber direction from primary motor area until the brain stem.

\section{References}

1. M. Naf, O. Kubler, R. Kikinis, M.E. Shenton, and G. Szekely. Characterization and Recognition of 3D Organ Shape in Medical Image Analysis Using Skeletonization. In IEEE/SIAM, editor, IEEE/SIAM Workshop on Mathematical Methods in Biomedical Image Analysis, 1996, pp. 139-150.

2. J.F. Mangin, J. Régis, and V. Frouin. Shape bottlenecks and conservative flow systems. In IEEE/SIAM, editor, IEEE/SIAM Workshop on Mathematical Methods in Biomedical Image Analysis, 1996, pp. 319-328.

3. D.C. Van Essen. A tension-based theory of morphogenesis and compact wiring in the central nervous system. Nature, vol. 385, 1997, pp. 313-318.

4. D. Le Bihan. Molecular Diffusion Nuclear Magnetic Resonance Imaging. Magnetic Resonance Quaterly, vol. 7, no. 1, 1991, pp. 1-30.

5. D. Le Bihan. Diffusion and Perfusion Magnetic Resonance Imaging, chapter A-2IV, pp. 50-57. Raven Press, Ltd., New-York, 1995.

6. C. Pierpaoli, P. Jezzard, P.J. Basser, A. Barnett, and G. Di Chiro. Diffusion Tensor MR Imaging of the Human Brain. Radiology, vol. 201, 1996, pp. 637-648.

7. J. Mattiello, P.J. Basser, and D. Le Bihan. The B Matrix in Diffusion Tensor Echo-Planar Imaging. Magnetic Resonance Magazine, vol. 37, 1997, pp. 292-300.

8. C. Pierpaoli and P.J. Basser. Toward a Quantitative Assessment of Diffusion Anisotropy. Magnetic Resonance Magazine, vol. 36, 1996, pp. 893-906.

9. S. Geman and D. Geman. Stochastic Relaxation, Gibbs Distributions, and the Bayesian Restoration of Images. IEEE PAMI, vol. 6, no. 6, 1984, pp. 721-741.

10. J. Besag. On the statistical analysis of dirty pictures. Journal of the Royal Statistical Society, Series B (Methodological), vol. 48, no. 3, 1986, pp. 259-302. 